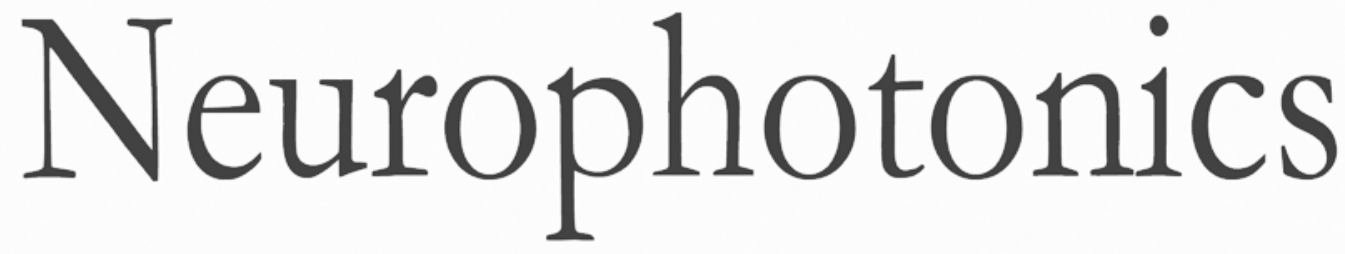

\title{
High-density speckle contrast optical tomography of cerebral blood flow response to functional stimuli in the rodent brain
}

Tanja Dragojević

Ernesto E. Vidal Rosas

Joseph L. Hollmann

Joseph P. Culver

Carles Justicia

Turgut Durduran 


\title{
High-density speckle contrast optical tomography of cerebral blood flow response to functional stimuli in the rodent brain
}

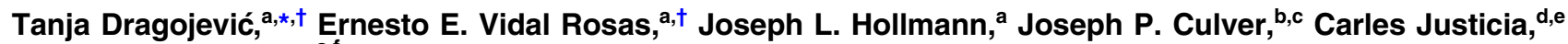 \\ and Turgut Durduran ${ }^{\mathrm{a}, \mathrm{f}}$ \\ aInstitut de Ciències Fotòniques, The Barcelona Institute of Science and Technology, Barcelona, Spain \\ bWashington University, School of Medicine, Department of Radiology, St. Louis, Missouri, United States \\ 'Washington University, Department of Physics, St. Louis, Missouri, United States \\ 'Institut d'Investigacions Biomèdiques de Barcelona, Consejo Superior de Investigaciones Científicas, Department of Brain Ischemia and \\ Neurodegeneration, Barcelona, Spain \\ eInstitut d'Investigacions Biomèdiques August Pi i Sunyer, Àrea de Neurociències, Barcelona, Spain \\ fInstitució Catalana de Recerca i Estudis Avançats, Barcelona, Spain
}

\begin{abstract}
Noninvasive, three-dimensional, and longitudinal imaging of cerebral blood flow (CBF) in small animal models and ultimately in humans has implications for fundamental research and clinical applications. It enables the study of phenomena such as brain development and learning and the effects of pathologies, with a clear vision for translation to humans. Speckle contrast optical tomography (SCOT) is an emerging optical method that aims to achieve this goal by directly measuring three-dimensional blood flow maps in deep tissue with a relatively inexpensive and simple system. High-density SCOT is developed to follow CBF changes in response to somatosensory cortex stimulation. Measurements are carried out through the intact skull on the rat brain. SCOT is able to follow individual trials in each brain hemisphere, where signal averaging resulted in comparable, cortical images to those of functional magnetic resonance images in spatial extent, location, and depth. Sham stimuli are utilized to demonstrate that the observed response is indeed due to local changes in the brain induced by forepaw stimulation. In developing and demonstrating the method, algorithms and analysis methods are developed. The results pave the way for longitudinal, nondestructive imaging in preclinical rodent models that can readily be translated to the human brain. () The Authors. Published by SPIE under a Creative Commons Attribution 4.0 Unported License. Distribution or reproduction of this work in whole or in part requires full attribution of the original publication, including its DOI. [DOI: 10.1117/1.NPh.6.4.045001]
\end{abstract}

Keywords: blood or tissue constituent monitoring; functional monitoring and imaging; medical and biological imaging; speckle imaging. Paper 19070R received Jul. 8, 2019; accepted for publication Sep. 12, 2019; published online Oct. 8, 2019.

\section{Introduction}

Microvascular cerebral blood flow (CBF) dynamics is an important biomarker of healthy brain development, pathology, and injury since it is a measure of the delivery of nutrients necessary for tissue metabolism and of the clearance of the by-products. The coupling between the neuronal activity and regional CBF is regarded as fundamental in the study of functional brain activity and is the basis of many functional neuroimaging techniques. ${ }^{1,2}$ For example, it is responsible for the blood oxygen level-dependent (BOLD) signal that is commonly employed in functional magnetic resonance imaging (fMRI). Wide-field superficial ${ }^{3}$ and deep tissue (>millimeters) diffuse optical ${ }^{4-6}$ methods provide similar contrasts, and in comparison to large imaging modalities, their advantages are portability, fast and continuous monitoring, the use of nonionizing radiation, and being noninvasive. The main contrast to optical methods has traditionally been that of the oxy- and deoxy-hemoglobin concentration ${ }^{7,8}$ but various methods utilizing laser speckle statistics have allowed for the direct measurement of blood flow. ${ }^{9-11}$

*Address all correspondence to Tanja Dragojević, E-mail: tanja.dragojevic@ icfo.es

†These authors have contributed equally.
Most common optical techniques for blood flow monitoring are based on the dynamic scattering of light and include laser Doppler flowmetry, ${ }^{12,13}$ laser speckle contrast imaging, ${ }^{9,11}$ and diffuse correlation spectroscopy (DCS). ${ }^{14}$ Of these methods, DCS uses long-coherence laser light with point sources and displaced point detectors to measure the temporal fluctuations of a diffuse speckle which is translated into a blood flow index (BFI) from the deep tissues using model-based algorithms. ${ }^{10}$ DCS is very attractive for $\mathrm{CBF}$ monitoring because its sensitivity can reach up to several centimeters. However, it also has several disadvantages, such as sophisticated hardware requirements and the associated lack of scalability thus reducing the spatial resolution and the ability to localize the signals. This is mainly because the high-density source-detector arrangements involving parallel acquisition from many source-detector pairs over a large range of separations are needed to achieve better resolution and location. This was, in fact, crucial to the success of diffuse optical tomography (DOT). ${ }^{15-17}$ This is not, so far, accessible for diffuse correlation tomography (DCT) ${ }^{18-20}$ To date, DCT has been limited to pilot studies on small animals ${ }^{18,19}$ and coarse measurements on humans. ${ }^{20}$

Recently, speckle contrast optical spectroscopy/tomography (SCOS/SCOT) was introduced to overcome some of these limitations. ${ }^{21-24}$ It combines single-point illumination with a two-dimensional detector array to probe deep tissues. This method has successfully been used to monitor relative cerebral 
blood flow (rCBF) due to changes in a functional task in the adult human brain. ${ }^{25}$ SCOT can achieve scalability using the speckle contrast with an appropriate correlation diffusion model to relax the requirement, which the data-type used by DCT has, i.e., relatively fast (tens of nanoseconds) output from the detectors, thus allowing for readily available detector arrays to be utilized in the measurements. ${ }^{25-28}$

Here we highlight speckle contrast optical tomography (SCOT) which, by taking advantage of the scalability of SCOS detection, achieves tomographic measurements using scanning sources and array detectors. ${ }^{27,28}$ Our recent developments in mice revealed comparable results to magnetic resonance imaging in following the infarct induced by $\mathrm{CBF}$ reduction. ${ }^{27} \mathrm{~A}$ similar development ${ }^{29}$ has been successfully used in the intraoperative room, where the three-dimensional (3-D) distribution of blood flow from a mastectomy skin flap was resolved. These applications highlight the potential of tomographic speckle contrast imaging in the clinic and in biomedical research. However, these examples are either too slow or use a too sparse array of detectors/sources to push the technique to its full potential.

On the other hand, traditional DOT has been pushed closer to its full potential by the introduction of high density (HD-DOT) and fast scanning. ${ }^{15,17,30}$ HD-DOT allows increased spatial resolution and improves the accuracy of the detected changes by utilizing many, densely spaced overlapping samples of the probed tissue volume. By being fast, it also allows for the monitoring of the evolution of the functional changes and the regression of the confounding systemic signals. HD-DOT was shown to approach the resolution and accuracy of BOLD-fMRI in the adult brain. ${ }^{31}$ However, HD-DOT measures the change in concentration of oxy- and deoxy-hemoglobin during functional activation in small animals and humans ${ }^{15,30,32}$ without retrieving $\mathrm{CBF}$ information. In HD-DOT, CBF information can be inferred by the use of specific challenges or contrast agents, thus making the measurements complex.

Given the above developments, the stage is now set for the introduction of fast, high-density SCOT (HD-SCOT) to achieve high-density 3-D blood flow imaging. The first step toward that end is to apply HD-SCOT in the rat brain, transcranially, without thinning the skull. This is motivated by the considerable interest in small animal imaging, since they allow the investigation of neurovascular coupling in the normal brain, during the progression of diseases (e.g., Alzheimer's) or severe pathological events (e.g., stroke). Furthermore, HD-SCOT would allow to follow up on the effects of therapeutic drugs, the evolution of treatment and the recovery of brain function ${ }^{33}$ over longer time periods (months to years). The measurement of the same parameter was considered by DCS in longitudinal monitoring of rodents, ${ }^{34}$ demonstrating that the growth of the animal can impact the measurements in a detrimental manner unless a large field-ofview (FOV) measurement with tomographic reconstruction is achieved. In other words, both the magnitude and the location of the signal changes over time in a rapidly aging small animal model.

In this study, we have developed and demonstrated the capabilities of SCOT for the monitoring of the cerebral blood flow response due to functional forepaw stimulation-induced changes in the somatosensory cortex of the rat brain. Tomographic images with good spatial resolution were possible due to the availability of high-density data. Furthermore, in order to increase the temporal resolution, we have introduced a technique based on a moving window scheme, which effectively improves the sampling efficiency.

\section{Materials and Methods}

\subsection{Animal Preparation}

The experimental protocol was approved by the ethical committee of the University of Barcelona. Adult male Wistar rats $(n=5)$ were anesthetized with $4 \%$ isoflurane and moved to a stereotaxic frame. The anesthesia was reduced to $2 \%$ isoflurane and the surgical procedure was performed, that consisted of the retraction of the skin and underlying muscle while keeping the skull intact, to avoid confounding effects. We note that this is not a necessary condition for the method and it could be applied fully noninvasively. ${ }^{35} \mathrm{~A}$ well surrounding the imaging area was built with dental cement and was filled with mineral oil to prevent the skull from drying out. After surgery, the isoflurane infusion was stopped and anesthesia was maintained with a continuous intravenous administration of $\alpha$-chloralose (40 mg/kg/h).

\subsection{Functional Activation Paradigm}

Electrical stimulation was achieved with a pair of subdermal needle electrodes that were inserted into right and left forepaws. The experiment consisted of electrically stimulating each forepaw individually with $200-\mu$ s pulses at $6-\mathrm{Hz}$ repetition rate and an amplitude of $2 \mathrm{~mA}$. The stimulation paradigm comprised of 18-s baseline, 30-s electrical stimulation, and 90-s recovery. This was repeated eight times for each forepaw. ${ }^{16}$ Once the functional protocol was completed, four repetitions of sham measurements were taken for the same duration, during which no electrical stimulation was performed, but the subdermal needles were kept inside forepaws.

\subsection{Magnetic Resonance Imaging}

To compare SCOT, functional MRI was acquired for the same animals $(n=5)$ under $\alpha$-chloralose $(40 \mathrm{mg} / \mathrm{kg} / \mathrm{h})$ anesthesia within 24 to $72 \mathrm{~h}$ after optical data were acquired. Experiments were conducted in a 7T MRI magnet (Bruker BioSpin, Germany). Coronal multislice spin-echo echo-planar images were acquired with a repetition time (TR) of $3 \mathrm{~s}$ and a bandwidth of $150 \mathrm{kHz}$. In total, five slices with $2-\mathrm{mm}$ thickness were acquired with an FOV of $39.9 \mathrm{~mm} \times 39.9 \mathrm{~mm}$ and a pixel size of $0.208 \mathrm{~mm} \times 0.208 \mathrm{~mm}$. Additionally, anatomical high-resolution T2-weighted images were obtained to align the fMRI and SCOT reconstructions (see Sec. 2.6). Anatomical data consisted of forty slices with $0.08-\mathrm{mm}$ thickness, FOV of $30 \mathrm{~mm} \times$ $30 \mathrm{~mm}$, and pixel size $0.117 \mathrm{~mm} \times 0.117 \mathrm{~mm}$.

\subsection{Speckle Contrast Optical Tomography Instrumentation}

The instrumentation was described in a previous publication ${ }^{27}$ and we review it in brief. The main components of the SCOT system are displayed in Fig. 1(a), where the scanning module consisted of a temperature-controlled continuous-wave laser at $785 \mathrm{~nm}$ (Thorlabs, Germany) that was focused by aspheric lenses onto a pair of galvo-mirrors (Nutfield), which were directing the light beam onto the rat head. ${ }^{27}$ The reflected diffuse light was captured by a CCD camera (Basler, Germany, scA640-120 fm). 


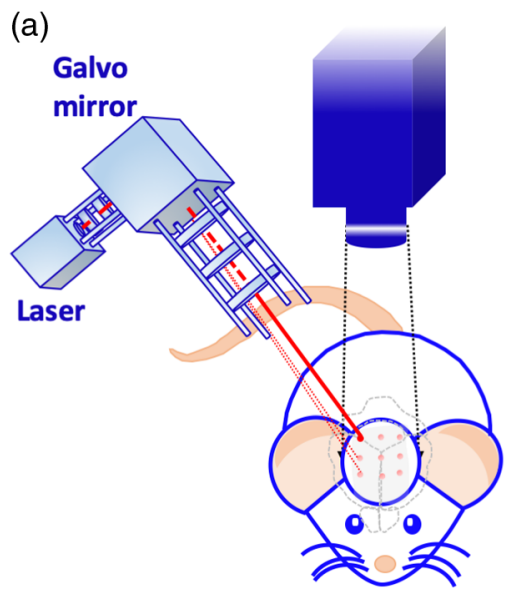

(b)

Fig. 1 (a) Schematic diagram of the main components of the scanning system. (b) The positions of the scanning sources. The central source was adjusted to match the location of the Bregma.

\subsection{Optical Data Acquisition Protocol}

Once the surgery was completed, the rat was moved under the imaging arm of the setup as shown in Fig. 1(a). During the alignment of the imaging domain, the central source of the array was adjusted to coincide with the location of a Bregma point [Fig. 1(b)]. The number of source positions was fixed at nine for all animals organized in a $3 \times 3$ arrays [Fig. 1(b)]. A calibration image was then taken where the physical imaged area per pixel was calibrated using a ruler placed on top of the rat's head. The $f$-number of the optical system was chosen to match the pixel to speckle size. Then the dental cement well was filled with mineral oil. Additionally, a green light LED (525 nm) was used to illuminate the rat's head and verify that the imaging area was free of contaminants (i.e., hair and bubbles). Otherwise, the mineral oil was withdrawn, the head was cleaned, and the oil was reapplied.

Furthermore, to obtain the baseline blood flow, 20 exposure times that were logarithmically spaced between $50 \mu \mathrm{s}$ and $10 \mathrm{~ms}$ were used to recover the baseline CBF.

Then the stimulation protocol (Sec. 2.2) was conducted while SCOT data were being acquired. Images were acquired with an exposure time of $1 \mathrm{~ms}$ and an FOV of $1.2 \mathrm{~cm} \times 1 \mathrm{~cm}$. One full tomographic data set consisted of the acquisition of a 100 images per source location, which was completed in $6 \mathrm{~s}$.

Calculation of the speckle contrast was done in the temporal approach. ${ }^{36}$ In the end, to account for the variance of the sensor dark noise, 900 images with an exposure time of $1 \mathrm{~ms}$ were collected with the laser light off.

\subsection{Theory and Algorithms}

\subsubsection{Theory of speckle contrast}

The speckle contrast squared $\kappa^{2}$ is defined as the ratio of the variance $\sigma^{2}$ to the squared mean value of the intensity $I^{2}$ as

$\kappa^{2}(\mathbf{r}, T)=\frac{\sigma^{2}(\mathbf{r}, T)}{I\langle(\mathbf{r}, T)\rangle^{2}}$,

where $T$ is the exposure time and $\mathbf{r}$ is the source-detector separation. The mean $I\langle(\mathbf{r}, T)\rangle^{2}$ and the variance can be computed either temporally (over independent speckles in a single pixel over time) or spatially (over independent speckles in different, neighboring pixels). ${ }^{36,37}$ Furthermore, the speckle contrast can be related to the normalized electric field autocorrelation function $g_{1}$ by

$\kappa^{2}(\mathbf{r}, T)=\frac{2 \beta}{T} \int_{0}^{T}\left(1-\frac{\tau}{T}\right)\left[g_{1}(\mathbf{r}, \tau)\right]^{2} \mathrm{~d} \tau$,

where $\tau$ is the correlation delay time, and $\beta$ is an optical system parameter proportional to the number modes/speckles in the detection system, which is $\sim 0.5$ for unpolarized light used in this study. This equation is related to the optical parameters of the medium and BFI through modeling of the $g_{1}$ function using the correlation diffusion equation. ${ }^{10}$ These methods were previously validated for SCOS/SCOT in phantoms, small animal models and adult, human subjects. ${ }^{21,28}$

\subsubsection{Speckle contrast correction and data analysis}

Prior to the solution of the inverse problem for the tomographic reconstruction, the speckle contrast was corrected to account for the shot and dark noise variations associated with the camera sensor. The corrected speckle contrast is defined as ${ }^{20}$

$\kappa_{c}^{2}(\mathbf{r}, T)=\frac{\sigma^{2}\left(I_{c}\right)-\sigma_{s}^{2}-\sigma_{d}^{2}}{\left\langle I_{c}\right\rangle^{2}}$

where $I_{c}=I-I_{d}$ is the dark corrected intensity, $I_{d}$ and $\sigma_{d}^{2}$ are the mean and variance of the dark noise over a number of frames, and $\sigma_{s}^{2}$ denotes the variance of the estimated shot noise. In addition, to improve the signal-to-noise ratio (SNR) and to reduce the computational load, the speckle contrast of four adjacent pixels was binned into a single pixel [Fig. 2(b)]. ${ }^{27}$

The baseline BFI was determined using multi-exposure speckle imaging (MESI), ${ }^{26,38}$ using the multi-exposure data that was acquired as described in Sec. 2.5 and Ref. 37. The MESI, together with Eq. (2), was used to fit for an averaged $\mathrm{BFI}_{0}$ using the dependence of the speckle contrast on the exposure time as previously described for SCOS. ${ }^{21,25,26}$

Valid source-detector pairs were selected based on two criteria, where first was that source-detector pairs with separations shorter than $0.15 \mathrm{~cm}$ were disregarded since the diffusion model fails to predict the transport of photon at short separations, ${ }^{39}$ 
(a)

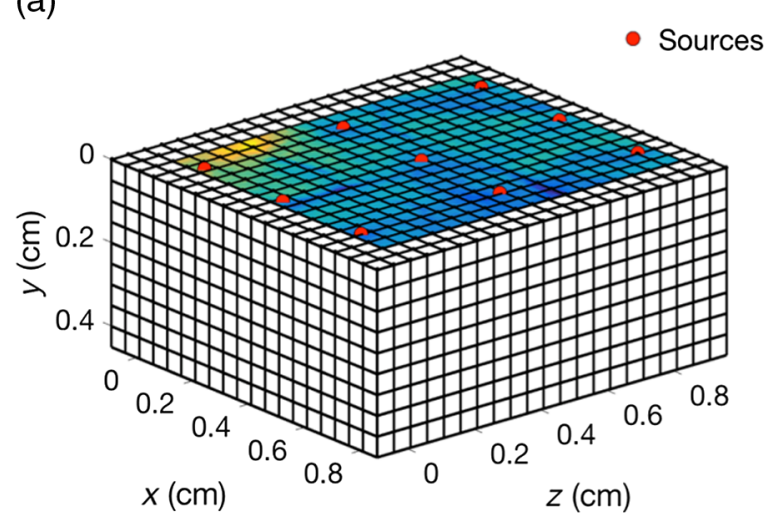

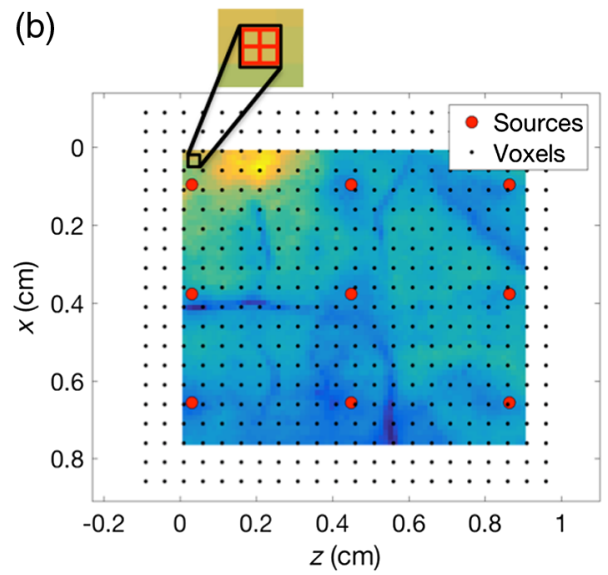

Fig. 2 (a) The imaging domain that was used for the tomographic reconstruction of $\triangle B F I$. (b) A 2-D schematic diagram showing the relative positions between sampled data, sources, and voxel positions. The black square illustrates the binning process where the speckle contrast of four adjacent pixels (red squares) is averaged to reduce the computational load.

also, pixels near the source illumination point were discarded since they were saturated. In the future, a higher order approximation to the radiation transport equation can be used to retain all the nonsaturated data. Second, source-detector pairs with a separation larger than $0.65 \mathrm{~cm}$ were discarded to avoid contamination or biasing of the signals due to the limited FOV, where many detectors were reaching the dental cement surrounding well and the lower SNR.

\subsubsection{Reconstruction of blood flow index changes}

Differential images of the BFI were obtained using the first Born approximation of Eq. $(2)^{28}$ at each time point. This is represented by

$\Delta \kappa_{c}^{2}(\mathbf{r}, T, t)=\kappa_{c}^{2}(t)-\kappa_{0, c}^{2}=\mathbf{J} \cdot \Delta \operatorname{BFI}(x, y, z, t)$,

where $\mathbf{J}$ is the Jacobian, $\kappa_{0, c}^{2}$ is the baseline (Sec. 2.6.4), corrected speckle contrast squared, and $\kappa_{c}^{2}$ is the corrected speckle contrast squared that was altered due to a perturbation. Under this approximation, the temporal changes in the measured speckle contrast are linearly related to spatial variations in the BFI $(\triangle \mathrm{BFI})$ in a $3-\mathrm{D}$ space. The tomographic image of these values can be obtained by inverting the above relationship. Since the inversion process is an ill-posed problem, we have regularized the inversion using a spatially varying Tikhonov regularization scheme. ${ }^{27,28,40}$

An illustration of the imaging domain is displayed in Fig. 2(a), which consisted of 5,280 voxels $(22 \times 20 \times 12)$. A sample image of the input measurement data is shown in Fig. 2(b) together with the source locations (red circles) and the voxel positions.

\subsubsection{Time series of relative cerebral blood flow}

Baseline data $\left(\kappa_{0, c}^{2}\right)$ were defined as the mean of the corrected speckle contrast during the first $18 \mathrm{~s}$. The time series of changes in the corrected speckle contrast $\Delta \kappa^{2}(t)$ from the baseline $\left(\kappa_{0, c}^{2}\right)$, i.e., $\Delta \kappa^{2}(t)=\kappa_{c}^{2}(t)-\kappa_{0, c}^{2}$, were inverted to obtain temporal changes of cerebral blood flow, i.e., $\Delta \mathrm{BFI}(t)$. The results are presented as relative temporal changes of $\mathrm{CBF}[\Delta \operatorname{rCBF}(t)]$, which is defined as the ratio of $\Delta \mathrm{BFI}(t)$ to the baseline BFI $\left(\mathrm{BFI}_{0}\right):{ }^{27}$

$\Delta \mathrm{rCBF}(t)=\frac{\Delta \mathrm{BFI}(t)}{\mathrm{BFI}_{0}} \times 100(\%)$.

To quantify the spatial extent of the activation at the depth with the largest change, the area of activation was defined as the number of pixels with $\mathrm{rCBF}>50 \%$ of the maximum response. ${ }^{11}$ Additionally, the recovered $\triangle \mathrm{rCBF}$ images were compared to the MRI data by co-registering the anatomical MRI and optical images using the Bregma point as a reference. Then optical data were transformed into the MRI coordinates taking the curvature of the brain into account for better visualization. ${ }^{27}$

\subsubsection{Sliding tomographic reconstruction scheme to improve the temporal resolution}

The standard reconstruction scheme, described in Fig. 3(a), involves solving the inverse problem once a full tomographic data set is available, i.e., after all sources have completed a scanning cycle. Then the process is repeated for subsequent scanning cycles. One disadvantage of this method is that rapidly evolving changes, such as those during somatosensory activation, of the signals are averaged over due to having a tomographic image every few seconds.

Here a reconstruction scheme based on a moving window was employed to improve the temporal resolution. It is similar to the jittering of the RT used in fMRI to improve the efficiency of the sampling. ${ }^{41}$ The process is displayed in Fig. 3(b), where a tomographic image can be obtained as soon as data from a new source position is available. This is possible on the basis that the reference source can be arbitrarily assigned and the necessary conditions for the inversion are fulfilled as long as data from every source is available [Fig. 3(b)]. Furthermore, since the time delay for switching between scanning positions is considerably smaller than the acquisition time, i.e., the scanning process can be considered continuous regardless of the selection of the source as a reference. The method does not produce independent samples, but rather a running average thus the reconstructions include data across different stages, Fig. 3(c) (dotted circles). 


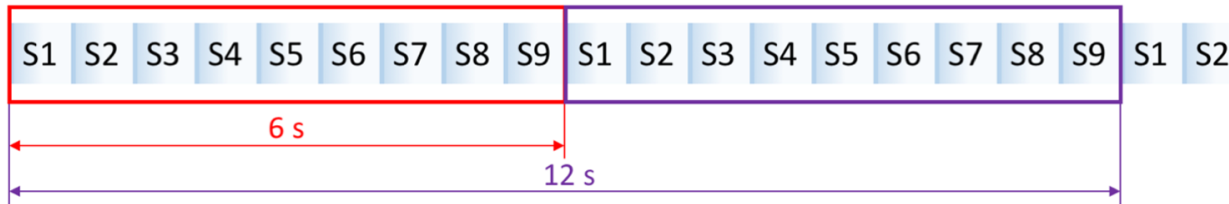

(b)

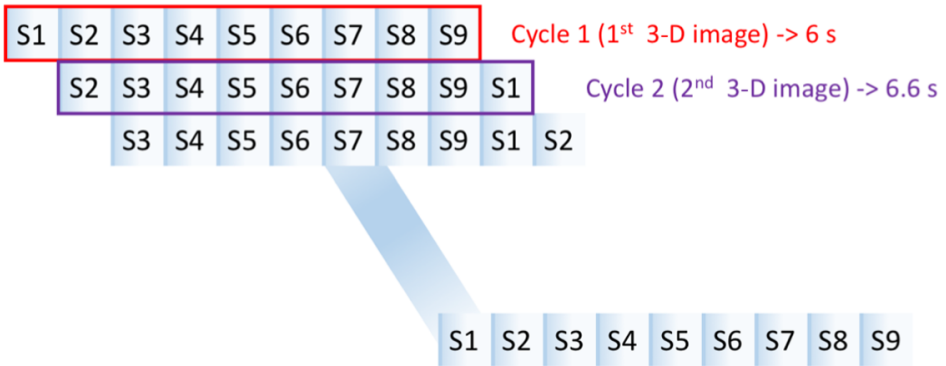

(c)

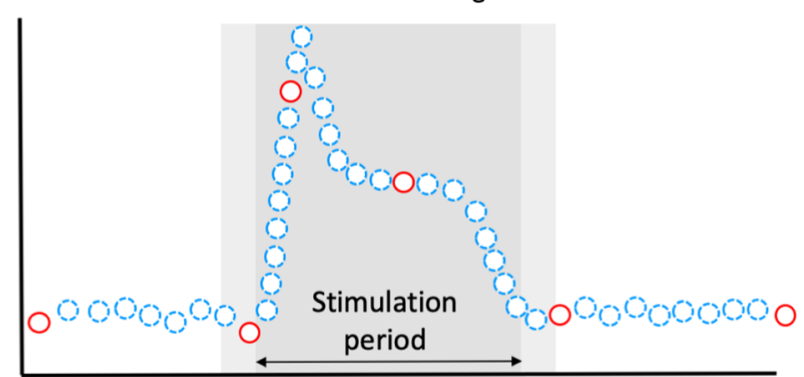

Fig. 3 (a) Standard reconstruction paradigm: a new 3-D image is produced after a full tomographic data set is available (every $6 \mathrm{~s}$ ). (b) Moving window reconstruction scheme: the first reconstruction takes $6 \mathrm{~s}$. However, a new 3-D image is produced as soon as data from a new source position is made available, which occurs approximately every $0.6 \mathrm{~s}$. (c) Comparison of the standard tomographic sampling against finer sampling achieved with the moving window tomographic sampling. The filled black circles (white) indicate the time points where a 3-D image is available with the standard scheme in contrast, the dotted void circles (blue) indicate the time points where a reconstructed image is available with the sliding window scheme. The shaded regions before and after the stimulus indicate the transition period where data from sources acquired after the stimulus onset contribute to $<50 \%$ of the reconstruction data.

In Fig. 3(c), the dense imaging achieved by the sliding window reconstruction scheme (void circles) is compared with the sparse temporal imaging obtained with the standard approach (filled circles). Since the "contamination" from samples, i.e., source positions, from different stages introduce artifacts due to the averaging of data at different conditions, we have also defined a period of influence to indicate the time points were the contribution of data from the stimulation stage is $<50 \%$. This period of influence is illustrated with the shaded regions before and after the stimulation stage in Fig. 3(c).

(a)

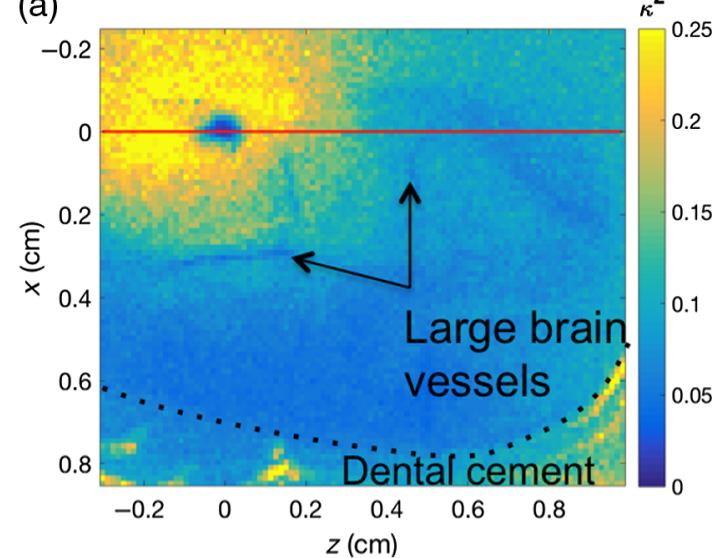

\section{Results}

\subsection{Data Conditioning Results}

Before image reconstruction, the usable source-detector pairs needed to be identified as described in Sec. 2.6.2. As Fig. 4(a) shows, the speckle contrast data from source one, which displays several features including the location of the source, large vessels and the effect of the dental cement. In particular, the speckle contrast along the horizontal red line is plotted in Fig. 4(b), where the effect of the source is visible.

Fig. 4 (a) Speckle contrast data from source one. (b) Speckle contrast squared as a function of the source-detector distance. The speckle contrast squared at the source location drops close to zero because these detectors were saturated where in this case, the variance is zero.

(b)

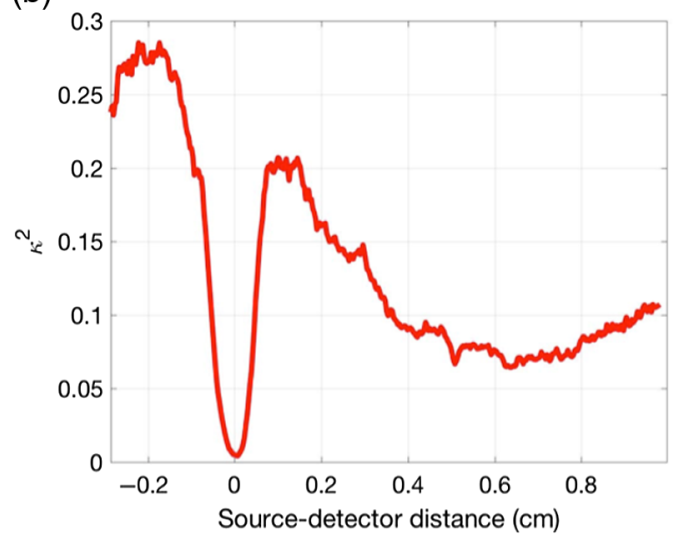


As described in Sec. 2.6.2, only source-detector pairs with separations in the range of 0.15 to $0.65 \mathrm{~cm}$ were kept before proceeding to the inversion process. On average, considering the nine sources, a total of $\sim 50,000$ valid source-detector pairs were used for image reconstruction for each animal.

\subsection{Differential Data and Image Reconstruction}

Figure 5(a) depicts a sample of the differential speckle contrast $\left(\Delta \kappa_{c}^{2}\right)$ during the stimulation stage from the source indicated with the red circle while the remaining source locations are shown in white. Particularly, there is a region indicated with the colored squares that shows a reduction of the speckle contrast during the stimulation stage. The relative temporal changes of $\Delta \kappa_{c}^{2}(t)$ with respect to baseline $\left(\kappa_{0, c}^{2}\right)$ at these locations are displayed in Fig. 5(b). A decrease of the speckle contrast during the stimulation can be observed followed by a return to the baseline. In other words, as expected, these changes reveal an increase of the CBF during the stimulation stage.

Figure 6(a) shows representative volumetric images of $\mathrm{rCBF}$ during left forepaw stimulation. Each column corresponds to different time points, whereas rows indicate slices in depth. The first column during the baseline period shows $\triangle \mathrm{rCBF}$ maps that are relatively homogeneous. However, during the stimulation stage, an active region at $y=-0.05 \mathrm{~cm}$ with higher $\Delta \mathrm{rCBF}$ is distinguishably followed by maps similar to those at baseline. The locations of apparent changes of $\triangle \mathrm{rCBF}$ at the skull level $(y=0 \mathrm{~cm})$ are due to the regularization operation which produces smooth responses rather than sharp changes. The time course of the activation region $(0.1 \mathrm{~cm} \times 0.1 \mathrm{~cm})$ centered at the peak value, displayed in Fig. 6(b), demonstrates the increased blood flow during the stimulation stage. The onset of the increase of $\triangle \mathrm{rCBF}$ before the start of the stimulation is an artifact produced by the sliding average effect, which covers data within the baseline and activation periods as described above.

\subsection{Comparison to Functional Magnetic Resonance Imaging}

The registration of the MRI and the optical data allowed a direct comparison of the location and the spatial extent of the activation. Figures 7(a) and 7(b) show representative BOLD fMRI and
$\Delta \mathrm{rCBF}$ images of an animal stimulated on the left forepaw. These images demonstrate that the activation is correctly located by speckle contrast. Furthermore, following the transformation of the recovered $\triangle \mathrm{rCBF}$ into the MRI coordinates, Fig. 7(c) demonstrates that the maximum change of $\triangle \mathrm{rCBF}$ occurs on the cortex region and not on the overlying skull. Similar images were obtained for the right forepaw stimulation [Figs. 7(d)-7(f)].

\subsection{Summary of Results over All Animals}

Figure 8 (a) shows the time courses of the changes in $\triangle \mathrm{rCBF}$ due to individual left forepaw stimulations for a representative animal at a depth $y=-0.05 \mathrm{~cm}$, which corresponds to the cortex layer. An initial overshoot at the beginning of the stimulation period is visible for all trials. Despite that the individual responses show considerable changes during the recovery period, the averaged response (black line) indicates that $\triangle \mathrm{rCBF}$ returns to baseline. Figure 8 (b) illustrates the $\triangle \mathrm{rCBF}$ due to left forepaw stimulation for each animal, where a consistent increase in $\triangle \mathrm{rCBF}$ during the activation period is visible across all animals. Figure $8(\mathrm{c})$ shows averaged $\triangle \mathrm{rCBF}$ over all five animals. A sharp increase in blood flow can be observed during the stimulation period. However, as mentioned previously (Sec. 3.2), the increase of $\Delta \mathrm{rCBF}$ starts before the stimulation. The period of influence of this artifact on the reconstruction is indicated with the shaded region outside the stimulation stage (black box). The corresponding sham responses for each animal are shown in Fig. 8(d), where no activation but flat responses can be seen during the stimulation period. Table 1 summarizes the estimated activation area for each individual at the slice with the maximum change.

\section{Discussion}

In this work, we have demonstrated three-dimensional monitoring of the cerebral blood flow response due to functional stimuli using high-density SCOT through the intact skull of the rat's head. The results show that the location and the spatial extent of the activation maps are in agreement with BOLD fMRI studies under a similar stimulation paradigm. ${ }^{42}$ Figure 6 (a) shows a clear distinction between the different stages of the experiment
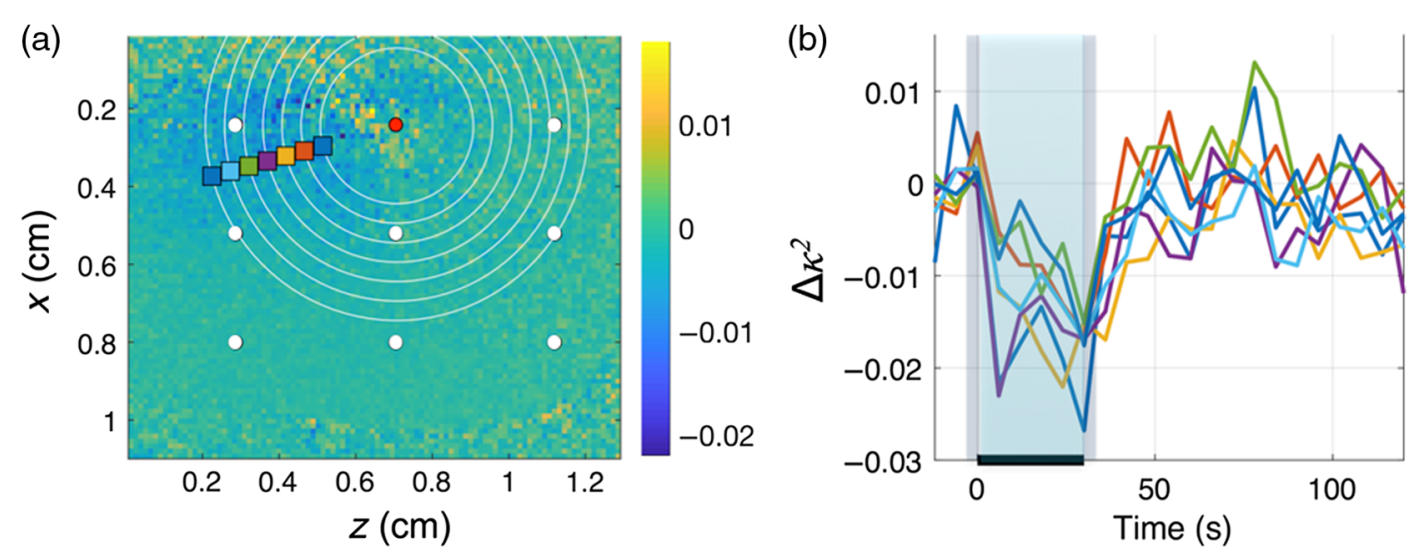

Fig. 5 (a) Representative differential speckle contrast data $\left(\Delta \kappa^{2}\right)$ during the simulation stage. (b) Time series of differential changes in speckle contrast $\Delta \kappa^{2}(t)$ at the locations indicated with the colored squares in (a). The relation with the spatial location is color-coded and the stimulation period is indicated with the shaded region. The shaded areas before and after the stimulation period correspond to the samples that produce artifacts in the reconstructed time series. 
(a)
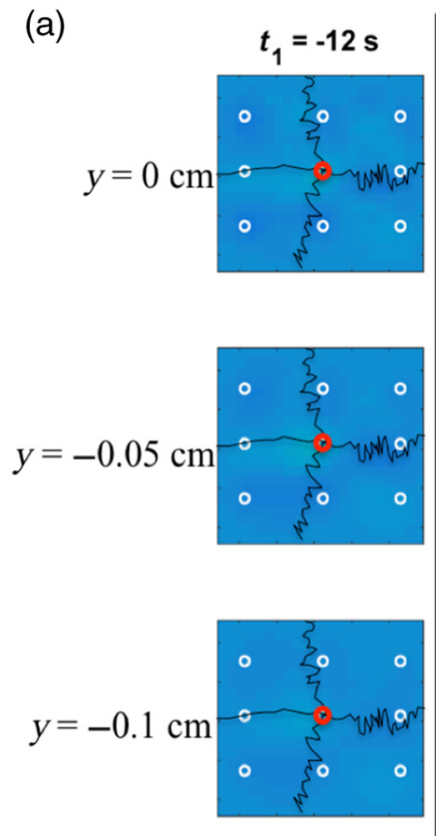

Sources Bregma
Stimulus

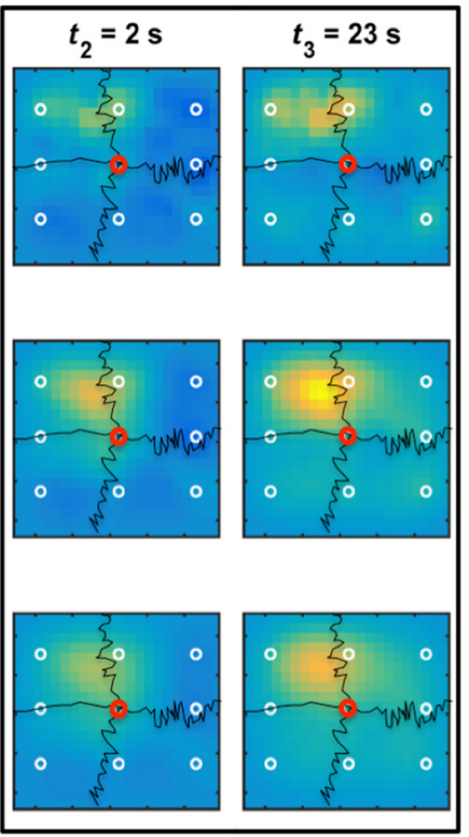

$\triangle \mathrm{rCBF}(\%)$
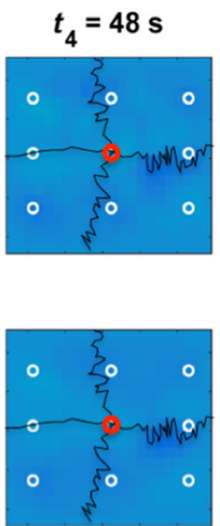

(b)

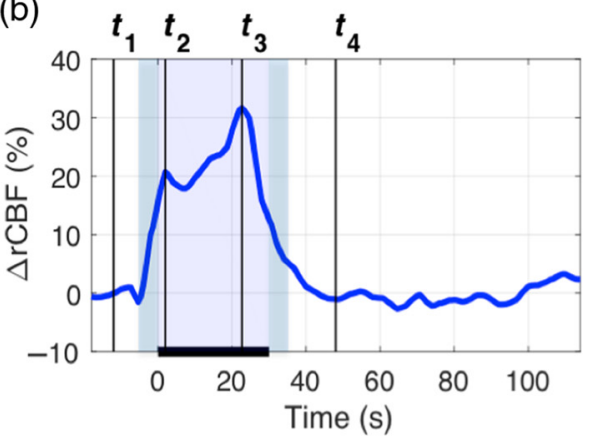

(c)

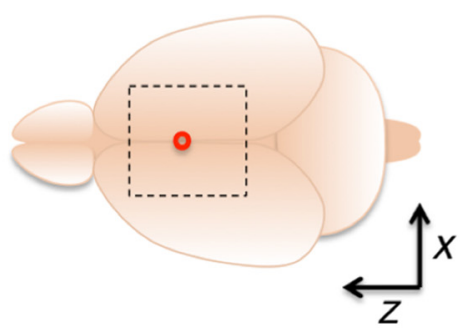

Fig. 6 (a) Tomographic imaging of rCBF for the left forepaw stimulation for representative animal. The maximum change is located at $y=-0.05 \mathrm{~cm}$ and not on the overlying skull $(y=0)$. (b) Temporal evolution of $\operatorname{rCBF}(y=-0.05 \mathrm{~cm})$ at baseline, stimulation, and recovery stages. (c) Reference illustration for orientation purposes.

and more importantly, the maximum change is correctly located at the cortex layer $(y=-0.05 \mathrm{~cm})^{42}$

The time trace of $\triangle \mathrm{rCBF}$ [Fig. 6(b)] shows a relatively rapid and sustained increase during the stimulation period. However, care must be taken to interpret the apparent increase before the start of the stimulation, which is introduced by the moving window reconstruction scheme. In the future, the temporal resolution can be improved by calculating the speckle contrast over space instead of time in sequential frames using a denser detection array structure while maintaining the high frame rate. If our method is further utilized, its effects can be minimized by considering an average of the forward and backward moving window, by explicitly accounting for the moving window using a weighing function in the postprocessing and by other methods

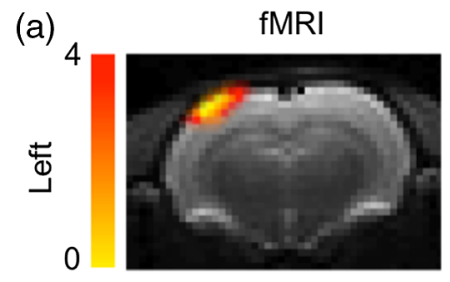

(b)

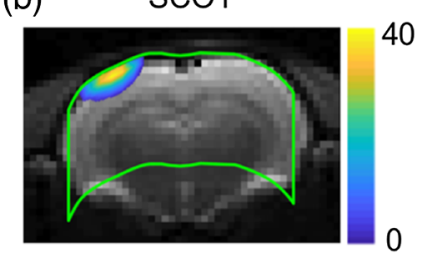

(d)

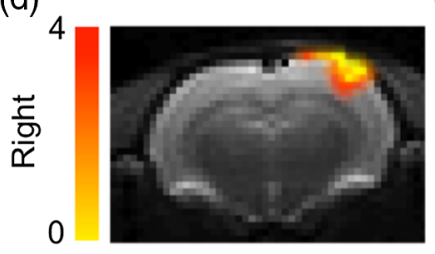

Bold $(\%)$ (e)

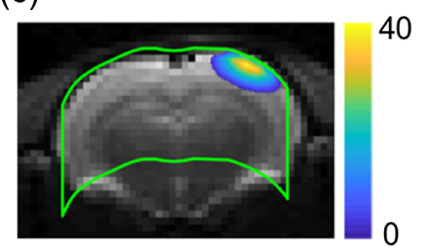

$\triangle \mathrm{rCBF}(\%)$

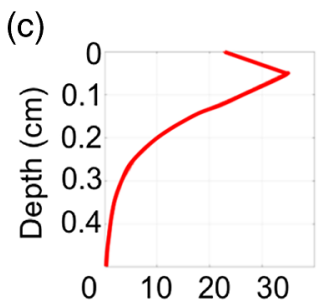

(f)

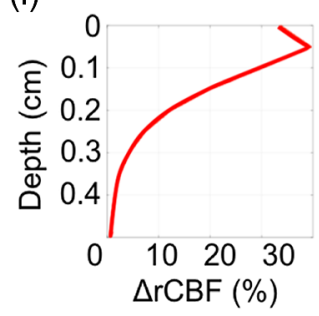

Fig. 7 (a) BOLD fMRI activation map during left forepaw stimulation. (b) $\Delta \mathrm{rCBF}$ map for $t=23 \mathrm{~s}$ overlaid onto the anatomical T2-weighted MRI image. The green line denotes the imaging domain used for the reconstruction. (c) $\triangle \mathrm{rCBF}$ as a function of depth. The corresponding images for right forepaw stimulation are displayed in panels (d) $-(\mathrm{f})$. 

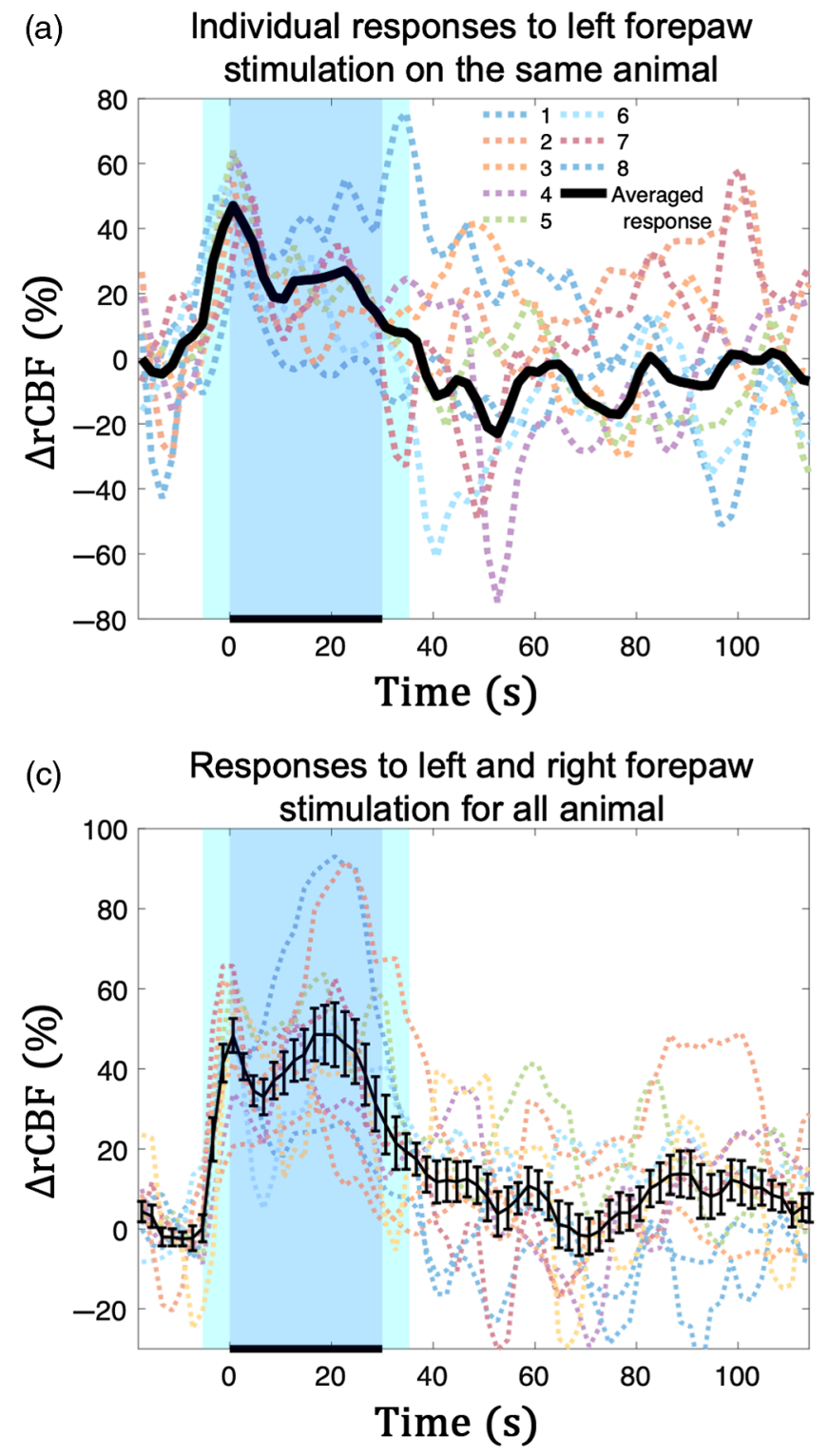

(b) Responses to left forepaw stimulation on each animal

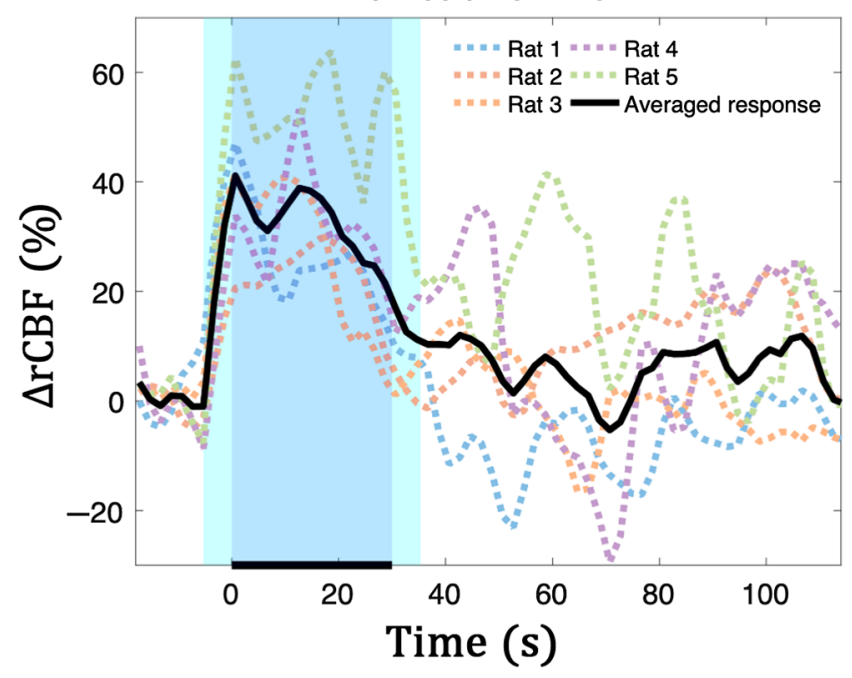

(d)

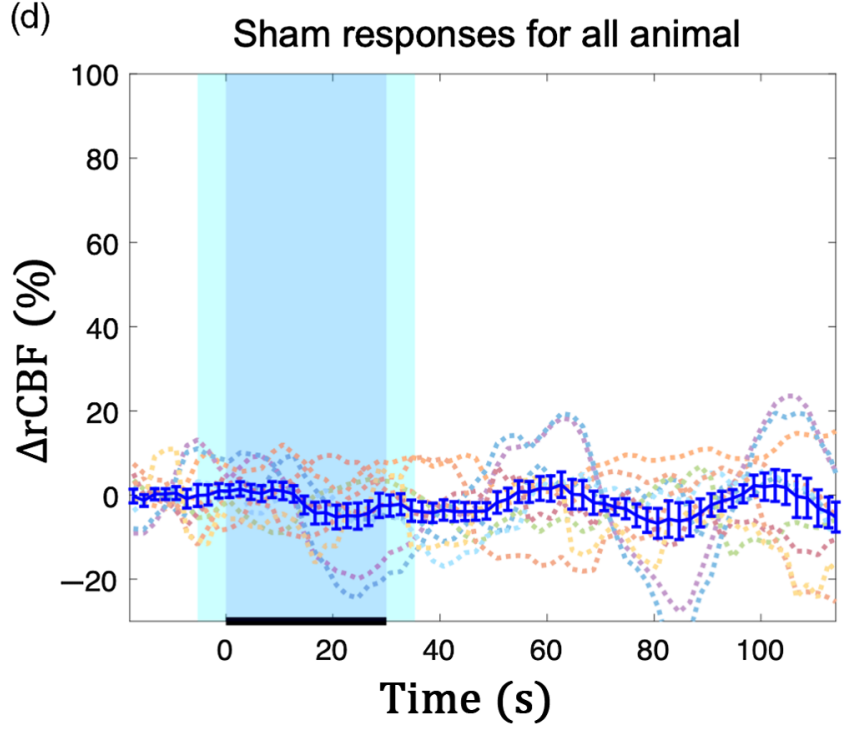

Fig. 8 (a) Series of plots of $\triangle \mathrm{rCBF}$ for a representative animal due to left forepaw stimulation. Each number denotes an individual trial, while the black line is the averaged response. (b) Time traces of mean, trial averaged $\triangle \mathrm{rCBF}$ for each animal during left forepaw stimulation, the black line represents the mean response over the animals. (c) Time traces of individual responses of $\triangle \mathrm{rCBF}$ due to separate left and right activation (forepaw stimulations). The black line corresponds to the grand average across all animals and stimuli. Similarly, panel (d) displays the sham response performed on the same animals.

Table 1 Results from individual rats for both left and right stimuli.

\begin{tabular}{lccc} 
& \multicolumn{3}{c}{ Activated area $\left(\mathrm{mm}^{2}\right)$} \\
\cline { 2 - 4 } Rat & Left & Right & Average \\
\hline 1 & 8.5 & 13.8 & 11.1 \\
2 & 17.8 & 10.8 & 14.3 \\
3 & 7.8 & 13.0 & 10.3 \\
4 & 7.5 & 13.0 & 10.3 \\
5 & 6.3 & 19.3 & 12.8 \\
\hline
\end{tabular}

that are utilized in similar windowing schemes. This could be the topic of further investigation in the future. In this study, we have opted for the current detector array for simplicity. In fact, speckle data can be acquired at even higher frame rates in animals ${ }^{43}$ and in adult humans ${ }^{44}$ that may in the future allow the consideration of the estimation of other biomarkers in addition to CBF. For example, with the improved temporal resolution, it would be possible to map the pulsatility of the cerebral blood flow due to cardiac cycle as a marker for vascular stiffness $^{43}$ and intracranial pressure. ${ }^{45}$

The spatial extent of the activation listed in Table 1 is in agreement with similar studies, ${ }^{11}$ and also with the size of the activation reported by other diffuse optical modalities, for example, the spatial extent obtained with SCOT is $11.7 \pm 1.7 \mathrm{~mm}^{2}$, which is very close to the activation area obtained with DOT 
which is $12.3 \pm 2 \mathrm{~mm}^{2} .{ }^{16}$ However, to show the volumetric change instead of the activation area, one would require a statistical analysis that would take into account factors such as differential effects or regularization at different slides and the hemodynamic response function. ${ }^{46}$ Figure 7 provides further confirmation of the accuracy of the SCOT technique in the location and the spatial extent of the activation. In particular, Figs. 7(c) and 7(f) display the maximum $\triangle \mathrm{rCBF}$ at the cortex and a decrease as the depth increases. However, the smooth and continuous decrease of $\Delta \mathrm{rCBF}$ at deeper layers $(y>0.15 \mathrm{~cm})$ is partly a consequence of the regularization method. The purpose of regularization is to stabilize the inversion process by finding a trade-off between the accuracy of the solution and the influence of noise, where this is achieved by attenuating the effect of higher-order modes, which result in smoother solutions. Additionally, it is well-known that recovered perturbations in the reflectance geometry tend to be located closer to the surface. ${ }^{40}$ Sharper changes can be obtained by adapting fully nonlinear iterative schemes which are readily available in open access software such as NIRFAST or TOAST for SCOT ${ }^{47,48}$ which would be the topic for further research.

SCOT provides a unique combination of deep tissue sensitivity together with a relatively high spatial resolution for the tomography of CBF changes. The possibility of high-density measurements contributed to an improvement in spatial resolution of $1 \mathrm{~mm}$. Similar developments in DOT ${ }^{49}$ provided substantial improvements in the spatial resolution and in the minimization of localization errors. High-density measurements allow the sampling of the brain tissue with a large number of overlapping measurements ${ }^{50}$ as a result, each point in the imaging space is better constrained and also less sensitive to noise during the inversion process. On the contrary, high-density data do not scale well in DCS/DCT because the monetary cost is prohibitively large since it requires expensive fast detectors for every added detector position. ${ }^{18,19,23}$

Figure 8 shows the performance of SCOT for the imaging of CBF in detail. As it is very typical of neuroimaging methods, intertrial, intra-animal, and inter-animal averaging were utilized. This was originally demonstrated for the measurement of CBF due to forepaw stimulation in Ref. 51, where they have characterized different sources of variability. In particular, Fig. 8(a) displays the individual responses for the left forepaw stimulation. The first point to note is that SCOT can characterize changes due to individual trials with an agreeable contrast and SNR. The variability is partly due to the variability introduced by the measurement method but also due to physiological variations in response to each trial including potential habituation effects. It is important to emphasize that the sharp increase in $\Delta \mathrm{rCBF}$ at the beginning of the stimulation period occurs in all trials and contributes to obtaining a robust peak in the averaged response (black line). Figure $8(\mathrm{~b})$ shows the $\triangle \mathrm{rCBF}$ after averaging over these trials for each animal again due to left forepaw stimulation. As expected, there are intra-animal variations presumably due to differences in anatomy, the dynamics of its physiology and condition at a given moment. However, the consistent initial overshoot followed by the persistent activation during the stimulation period, observed across all animals, demonstrates that SCOT is capable of imaging CBF in a robust manner. This characteristic is further supported by Fig. 8(c), where the grand average is plotted against the individual responses for the five animals and from the stimulation of both hemispheres. Baseline, activation, and recovery stages are markedly distinguishable from each other. For comparison, Fig. 8(d) shows the sham responses of the same animals, where mainly flat responses characterized the trends and allow one to appreciate the repeatability of the method. We note that a Wilcoxon test has demonstrated that this response reaches statistical significance in all animals during stimuli but not during sham. However, a full-fledged statistical analysis using a general linear model is beyond the scope of this work. Taken together, Figs. 8(a)-8(d) demonstrate the feasibility of tomographic imaging of changes in $\triangle \mathrm{rCBF}$ from a single trial level to a population level, with a considerable improvement of the response due to the effect of averaging. This is not always true for all neuroimaging methods.

Future works using multimodal approaches, e.g., combining DOT with SCOT and/or different fMRI methods with SCOT can be envisioned. These methods would enable a quantitative comparison of the $\mathrm{CBF}$ response to that of the oxygenation and derive the changes in cerebral metabolic rate of oxygen extraction. We have not carried this out in this manuscript since the fMRI data were not taken simultaneously and the protocol was not optimized for this purpose.

As partially demonstrated in this work, SCOT allows for a range of advantages over traditional DCT. SCOT can be implemented with arrays of detectors using existing technologies, which, as mentioned in Sec. 1 and Refs. 22-24, 27-29, allows for the improvement of the SNR, FOV, implementation of high-density source-detector pairs, and for potentially low cost, wearable devices. ${ }^{25}$ The simplicity of the data-type allows for embedding the calculations in firmware, ${ }^{25}$ which, in turn, allows for robust devices that could be utilized in the field without relying on fragile software and computers. The usability of cameras $^{24,27,29}$ and camera-like arrays ${ }^{30,35}$ readily enable noncontact scanning which can bring substantial benefits to imaging. For example, it allows the collection of highly dense data at multiple locations with lesser mechanical limitations such as those due to the location and the physical diameter of the fiber cladding. ${ }^{30}$ However, noncontact measurements have limitations and we note here, that contact measurements can be implemented with SCOT, ${ }^{30,52}$ which would allow for measurements through the hair when translating to studies on humans. ${ }^{30}$ Other implementations can also be envisioned combining spatial, spectral, and temporal information efficiently.

Our previous study demonstrated the possibility of tomographic brain imaging of stroke in a mouse model, ${ }^{27}$ where the present work is an extension to a larger geometry, to deeper and more rapidly evolving signals and to a more challenging experimental paradigm. Combining both contributions, this technology provides new opportunities for the study of the rodent brain after an injury. For example, it would be possible to monitor not only pathological changes in the brain due to a stroke, but also to study the evolution of the functional brain activity after the injury ${ }^{51,53,54}$ and in the long term, it would also be possible to study longitudinal effects ${ }^{17,30,34}$ such as those due to rehabilitation. The unique innovation of HD-SCOT is that these studies can be carried out with the same imaging platform in a noninvasive manner. The unique innovation of HD-SCOT is that these analyses can be carried out with the same imaging platform.

\section{Conclusion}

The feasibility of functional brain monitoring using high-density SCOT has been demonstrated. Robust changes of $\triangle \mathrm{rCBF}$ due to electrical stimulation of forepaw have been observed and the 
spatial extent, location, and magnitude of changes are in agreement with results reported in fMRI studies and other optical imaging modalities.

\section{Disclosures}

E. E. Vidal Rosas, T. Dragojević, J. L. Hollmann, and C. Justicia declare no conflict of interest, financial or otherwise. $T$. Durduran and J. P. Culver are inventors on relevant patents.

\section{Acknowledgments}

This project was funded by Fundació CELLEX Barcelona, Ministerio de Economía y Competitividad/FEDER (PHOTODEMENTIA, DPI2015-64358-C2-1-R), Instituto de Salud Carlos III/FEDER (MEDPHOTAGE, DTS16/00087), the "Severo Ochoa" Program for Centers of Excellence in $\mathrm{R} \backslash \& D$ (SEV-2015-0522), the Obra Social "la Caixa" Foundation (LlumMedBcn), AGAUR-Generalitat (2017 SGR 1380), LASERLAB-EUROPE IV, and "Fundació La Marató TV3." We acknowledge useful discussions with Dr. Hari Varma and Dr. Claudia Valdes.

\section{References}

1. A. Devor et al., "Frontiers in optical imaging of cerebral blood flow and metabolism," J. Cereb. Blood Flow Metab. 32(7), 1259-1276 (2012).

2. A. Villringer and U. Dirnagl, "Coupling of brain activity and cerebral blood flow: basis of functional neuroimaging," Cerebrovasc. Brain Metab. Rev. 7(3), 240-276 (1995).

3. A. Grinvald et al., "Functional architecture of cortex revealed by optical imaging of intrinsic signals," Nature 324(6095), 361-364 (1986).

4. A. Gibson and H. Dehghani, "Diffuse optical imaging.," Philos. Trans. A. Math. Phys. Eng. Sci. 367(1900), 3055-3072 (2009).

5. S. Fantini, B. Frederick, and A. Sassaroli, "Perspective: prospects of non-invasive sensing of the human brain with diffuse optical imaging," APL Photonics 3(11), 110901 (2018).

6. H. Obrig, "NIRS in clinical neurology-a 'promising' tool?" Neuroimage 85, 535-546 (2014).

7. Y. Hoshi, "Functional near-infrared optical imaging: utility and limitations in human brain mapping," Psychophysiology 40(4), 511-520 (2003).

8. M. Ferrari, L. Mottola, and V. Quaresima, "Principles, techniques, and limitations of near infrared spectroscopy," Can. J. Appl. Physiol. 29(4), 463-487 (2004)

9. A. K. Dunn et al., "Dynamic imaging of cerebral blood flow using laser speckle," J. Cereb. Blood Flow Metab. 21(3), 195-201 (2001).

10. T. Durduran et al., "Diffuse optics for tissue monitoring and tomography," Rep. Prog. Phys. 73(7), 076701 (2010).

11. T. Durduran et al., "Spatiotemporal quantification of cerebral blood flow during functional activation in rat somatosensory cortex using laserspeckle flowmetry," J. Cereb. Blood Flow Metab. 24, 518-525 (2004).

12. R. Bonner and R. Nossal, "Model for laser Doppler measurements of blood flow in tissue," Appl. Opt. 20(12), 2097-2107 (1981).

13. U. Lindauer, A. Villringer, and U. Dirnagl, "Characterization of CBF response to somatosensory stimulation: model and influence of anesthetics," Am. J. Physiol. 264(4), H1223-H1228 (1993).

14. D. A. Boas and Y. Campbell, "Scattering and imaging with diffusing temporal field correlations," Phys. Rev. Lett. 75, 1855-1858 (1995).

15. J. P. Culver et al., "Volumetric diffuse optical tomography of brain activity," Opt. Lett. 28(21), 2061-2063 (2003).

16. J. P. Culver et al., "Evidence that cerebral blood volume can provide brain activation maps with better spatial resolution than deoxygenated hemoglobin," Neuroimage 27, 947-959 (2005).

17. M. D. Wheelock, J. P. Culver, and A. T. Eggebrecht, "High-density diffuse optical tomography for imaging human brain function," Rev. Sci. Instrum. 90(5), 051101 (2019).

18. S. Han et al., "Temporal blood flow changes measured by diffuse correlation tomography predict murine femoral graft healing," PLoS One 13(5), e0197031 2018.
19. C. Zhou et al., "Diffuse optical correlation tomography of cerebral blood flow during cortical spreading depression in rat brain," Opt. Express 14(3), 1125-1144 (2006).

20. L. He et al., "Noncontact diffuse correlation tomography of human breast tumor," J. Biomed. Opt. 20(8), 086003 (2015).

21. C. P. Valdes et al., "Speckle contrast optical spectroscopy, a noninvasive, diffuse optical method for measuring microvascular blood flow in tissue," Biomed. Opt. Express 5(8), 2769-2784 (2014).

22. R. Bi et al., "Optical methods for blood perfusion measurement-theoretical comparison among four different modalities," J. Opt. Soc. Am. A 32(5), 860-866 (2015).

23. Y. Lin et al., "Three-dimensional flow contrast imaging of deep tissue using noncontact diffuse correlation tomography," Appl. Phys. Lett. 104(12), 121103 (2014).

24. R. Bi, J. Dong, and K. Lee, "Deep tissue flowmetry based on diffuse speckle contrast analysis," Opt. Lett. 38(9), 1401-1403 (2013).

25. T. Dragojević et al., "Compact, multi-exposure speckle contrast optical spectroscopy (SCOS) device for measuring deep tissue blood flow," Biomed. Opt. Express 9(1), 322-334 (2018).

26. T. Dragojević et al., "High-speed multi-exposure laser speckle contrast imaging with a single-photon counting camera," Biomed. Opt. Express 6(8), 2865-2876 (2015).

27. T. Dragojević et al., "High-density speckle contrast optical tomography (SCOT) for three-dimensional tomographic imaging of the small animal brain," NeuroImage 153, 283-292 (2017).

28. H. M. Varma et al., "Speckle contrast optical tomography: a new method for deep tissue three-dimensional tomography of blood flow," Biomed. Opt. Express 5(4), 1275-1289 (2014).

29. C. Huang et al., "Noncontact 3-D speckle contrast diffuse correlation tomography of tissue blood flow distribution," IEEE Trans. Med. Imaging 36(10), 2068-2076 (2017).

30. A. T. Eggebrecht et al., "Mapping distributed brain function and networks with diffuse optical tomography," Nat. Photonics 8(6), 448-454 (2014).

31. A. T. Eggebrecht et al., "A quantative spatial comparison of highdensity diffuse optical tomography and fMRI cortical mapping," Neuroimage 61(4), 1120-1128 (2012).

32. Z. J. Lin et al., "Volumetric diffuse optical tomography for small animals using a CCD-camera-based imaging system," Int. J. Opt. 2012, $1-10(2012)$.

33. U. Dirnagl, C. Iadecola, and M. A. Moskowitz, "Pathobiology of ischaemic stroke: an integrated view," Trends Neurosci. 22(9), 391397 (1999).

34. I. Blanco et al., "Longitudinal, transcranial measurement of functional activation in the rat brain by diffuse correlation spectroscopy," Neurophotonics 4(4), 045006 (2017).

35. C. Huang et al., "Noninvasive noncontact speckle contrast diffuse correlation tomography of cerebral blood flow in rats," Neuroimage 198, 160-169 (2019).

36. D. A. Boas and A. K. Dunn, "Laser speckle contrast imaging in biomedical optics," J. Biomed. Opt. 15(February), 011109 (2010).

37. R. Bandyopadhyay et al., "Speckle-visibility spectroscopy: a tool to study time-varying dynamics," Rev. Sci. Instrum. 76(9), 093110 (2005).

38. A. B. Parthasarathy et al., "Robust flow measurement with multiexposure speckle imaging," Opt. Express 16(3), 1975-1989 (2008).

39. P. D. Kaplan et al., "Geometric constraints for the design of diffusingwave spectroscopy experiments," Appl. Opt. 32(21), 3828-3836 (1993).

40. B. R. White, "Developing high-density diffuse optical tomography for neuroimaging," PhD Thesis, p. 173 (2012).

41. M. Watanabe et al., "Temporal jitter of the BOLD signal reveals a reliable initial dip and improved spatial resolution," Curr. Biol. 23(21), 2146-2150 (2013).

42. J. B. Mandeville and J. J. A. Marota, "Vascular filters of functional MRI: spatial localization using BOLD and CBV contrast," Magn. Reson. Med. 42(3), 591-598 (1999).

43. D. D. Postnov et al., "Cardiac pulsatility mapping and vessel type identification using laser speckle contrast imaging," Biomed. Opt. Express 9(12), 6388-6397 (2018).

44. T. Dragojevíc et al., "High-density functional speckle contrast optical tomography (HD-fSCOT) of the adult brain," in ECBO (2019).

45. D. Wang et al., "Fast blood flow monitoring in deep tissues with real-time software correlators," Biomed. Opt. Express 7(3), 776 (2016). 
46. F. Scholkmann et al., "A review on continuous wave functional nearinfrared spectroscopy and imaging instrumentation and methodology," Neuroimage 85(Pt 1), 6-27 (2014).

47. H. Dehghani et al., "Near infrared optical tomography using NIRFAST: algorithm for numerical model and image reconstruction," Commun. Num. Methods Eng. 25(6), 711-732 (2008).

48. M. Schweiger and S. Arridge, "The Toast++ software suite for forward and inverse modeling in optical tomography," J. Biomed. Opt. 19(4), 040801 (2014).

49. B. R. White and J. P. Culver, "Quantitative evaluation of high-density diffuse optical tomography: in vivo resolution and mapping performance," J. Biomed. Opt. 15(April), 026006 (2010).

50. Y. Zhan et al., "Image quality analysis of high-density diffuse optical tomography incorporating a subject-specific head model," Front. Neuroenerg. 4, 6 (2012).

51. J. A. Detre et al., "Signal averaged laser Doppler measurements of activation-flow coupling in the rat forepaw somatosensory cortex," Brain Res. 796(1-2), 91-98 (1998).

52. K. M. Bergonzi et al., "Lightweight sCMOS-based high-density diffuse optical tomography," Neurophotonics 5(3), 035006 (2018).

53. W. B. Baker et al., "Noninvasive optical monitoring of critical closing pressure and arteriole compliance in human subjects," J. Cereb. Blood Flow Metab. 37(8), 2691-2705 (2017).

54. J. Luckl et al., "Characterization of periinfarct flow transients with laser speckle and Doppler after middle cerebral artery occlusion in the rat," J. Neurosci. Res. 87, 1219-1229 (2009).

Tanja Dragojević received her PhD from ICFO-The Institue of Photonic Sciences, Spain, in the Medical Optics Group. Her research was focused on developing novel speckle based intrumentation and protocols, for preclinical and clinical studies. Currently she is working as a research engineer in the Medical Optics Group on development of speckle based intrumentation for clinical and industrial applications.

Ernesto E. Vidal Rosas is a research associate in the Department of Medical Physics and Biomedical Engineering at UCL. He obtained a
$\mathrm{PhD}$ in control engineering at the University of Sheffield, followed by a postdoctoral position at ICFO-The Institue of Photonic Sciences, Spain. Currently, his work is focused on the development of reconstruction algorithms for high-density diffuse optical tomography (HD-DOT). His research interests also include speckle imaging, diffuse correlation spectroscopy/tomography, broad-band spectroscopy and system identification.

Joseph L. Hollmann obtained his PhD from Northeastern University, in 2014, worked as a postdoctoral fellow at ICFO-The Institute of Photonic Sciences-and moved onto industry afterwards. His research interests cover a large variety of topics ranging from laser speckles in turbid media to others.

Joseph P. Culver is a professor of radiology at Washinton University. He obtained a PhD in physics at the University of Pennsylvania for work in ultrafast infrared laser spectroscopy. As a postdoc, he developed diffuse optical tomography systems for imaging breast cancer and imaging cerebal hemodynamics in animals. His lab explores ways of leveraging optical measurements for neuroimaging, with a focus on development of diffuse optical tomography techniques for mapping human brain function.

Carles Justicia received his $\mathrm{PhD}$ in biology from the University of Barcelona in 2001, working on death and survival pathways activated after stroke. He specialized in MR imaging at the Max Planck Institute for Neurological Research in Cologne. He is currently a senior researcher at the Biomedical Research Institute in Barcelona (IIBBCSIC) and is part of the group "Brain ischemia: Clinical and experimental studies" at IDIBAPS, where he investigates vascular changes associated with stroke and aging.

Turgut Durduran is an ICREA professor at ICFO-The Institute of Photonic Sciences (Spain)-where he leads the Medical Optics Group. His research interests revolve around the use of diffuse light to noninvasively probe tissue function. The group develops new technologies and algorithms and routinely translates them to preclinical, clinical, and industrial applications. 